\title{
Interaction Between CCL18 and GPR30 Differs from the Interaction Between Estradiol and GPR30
}

\author{
ROLAND SCHMIDT-WOLF ${ }^{1}$ and GERNOT ZISSEL ${ }^{2}$ \\ ${ }^{1}$ Department of Anesthesiology and Intensive Care Medicine, University Hospital Bonn, Bonn, Germany; \\ ${ }^{2}$ Department of Pneumology, Medical Center - University of Freiburg, \\ Faculty of Medicine, University of Freiburg, Freiburg, Germany
}

\begin{abstract}
Background/Aim: $C$-C motif chemokine ligand 18 (CCL18) is overexpressed in the microenvironment of tumors, promotes invasion and metastasis and is thus important for the therapeutic outcome of many tumor entities. The Gs-coupled seven-transmembrane receptor GPR3O is known as both a CCL18 and an estrogen receptor; its activation by estradiol leads to a transactivation of membrane-tethered pro-heparin-binding EGF-like growth factor and the MAPK/ERK pathway. We examined whether this signaling pathway remains the same under CCL18 stimulation, as opposed to estradiol stimulation. Materials and Methods: We investigated the effects of CCL18 on the lung cancer cell line A549, that show low GPR30 expression and the breast cancer cell lines MCF-7, that has high GPR30 expression and MDA-MB-231. These cells were stimulated in different media with CCL18 and then analyzed by qPCR, In-Cell Western ${ }^{\circledR}$, western blot and ELISA. Results: Many similarities on the effect of CCL18 on the already known estradiol-activated signaling pathway via the $G$ protein-coupled estrogen receptor GPR30 were identified. GPR3O is involved in the expression of matrix metalloproteinases (MMPS), which may play a role in the transactivation of ERK-1/-2 via the cleavage of membranebound $H B-E G F$, via Src-related tyrosine kinases and $G \beta \gamma$ subunits. With increasing CCL18 concentration, the expression of MMP7 decreased in A549 cells. With decreasing estrogen content of the medium, there was an
\end{abstract}

This article is freely accessible online.

Correspondence to: Dr. Roland Schmidt-Wolf, Department of Anesthesiology and Intensive Care Medicine, University Hospital Bonn, Venusberg-Campus 1, 53127 Bonn, Germany. Tel: +49 15738111716, Fax: +49 22828717065, e-mail: roland.schmidtwolf@ukbonn.de

Key Words: Ccl18 protein, gpr30, epithelial-mesenchymal transition (EMT), cancer, metastasis. increasing effect of CCL18 on the inhibition of the relative expression of MMP7. Inhibition of GPR30 with G15 also resulted in a decrease in the relative expression of $M M P 7$, irrespective of the subsequent stimulation with CCL18. This is a rather unexpected result, because the estrogen estradiol and CCL18 both activate GPR3O. MCF-7 cells which express more GPR30 did not show any dependence of the relative MMP7 expression on CCL18 except in estrogen-free FCS medium. CCL18 induced an increased relative ERK activation in In-Cell western (ICW) at A549 cells. Stimulation with CCL18 caused decreased ERK activation with simultaneous inhibition of adenylate cyclase in MCF-7. However, stimulation with CCL18 and simultaneous inhibition of cyclooxygenase in MCF-7 resulted in increased ERK activation. In A549, stimulation with CCL18 and coincubation with dbcAMP resulted in decreased ERK activation in both ICW and Western blot. Conclusion: In summary, the Gs-coupled receptor GPR30 plays an important role in the signaling pathway of CCL18. CCL18 and estradiol may not lead to the same signaling pathway after activating GPR3O.

Over the last few decades, there has been a significant increase in the prevalence and incidence of many tumor diseases in Germany and globally (1). At the same time, a better understanding of oncogenesis and the development of targeted cancer therapies in combination with conventional therapies have led to improved outcomes in cancer treatment (2). Tumor cells are characterized by a change in the expression of their surface receptors. The overexpression of certain receptors compared to healthy cells makes targeted cancer therapy possible. For example, the antibody trastuzumab has been successfully used in human epidermal growth factor receptor 2 (HER2) positive breast cancer $(3,4)$. We examined lung and breast cancer cell lines because both are derived from very common diseases. Lung cancer is currently the leading cause of cancer death worldwide in both men and women (5). Breast cancer is the most common cancer in women in Europe and the US (6). Chemotactic cytokines are known to act as 
messengers in the development, metastasis and angiogenesis of tumors $(7,8)$. However, their role in metastasis is poorly understood. When tumor cells metastasize and during tumor invasion, epithelial-to-mesenchymal transition (EMT) is required. EMT is an important process in embryogenesis and wound healing. During this process formerly epithelial cells lose their cell polarity and gain migratory properties (9). Our group previously found that chemokine (C-C motif) ligand 18 (CCL18) induces EMT in the lung cancer cell line A549, which promotes metastasis and resistance to chemotherapy (7). A recent study found that CCL18 promotes the invasion and the metastasis of breast cancer through the pleiotropic protein Annexin A2 by EMT through the PI3K/Akt/GSK3 $\beta /$ Snail signaling pathway (10). CCL18 is also referred to as PARC (pulmonary and activation-regulated chemokine), DC-CK-1 (dendritic cell chemokine 1) or AMAC-1 (alternative macrophage activation-associated CC chemokine-1), among others (8). In vivo the homeostatic chemokine CCL18 appears to exist only in primates and is expressed and secreted in high constitutive concentrations in serum by alternatively activated macrophages (M2) and dendritic cells $(3,11)$. Catusse et al. identified the G-protein coupled receptor GPR30 as a CCL18 receptor (3), which is also referred to as G-protein coupled estrogen receptor GPER. GPR30 expression correlates with Her-2/neu expression in breast cancer, whereas $\mathrm{ER} \alpha / \beta$ correlates inverse with Her-2/neu expression $(12,13)$.

Filardo et al. showed that estrogen activates GPR30 (1416). Since estrogen and CCL18 both activate GPR30 as a common receptor, we tried to identify whether CCL18initiated GPR30 activation occurs via the same signaling pathway as estrogen-initiated GPR30 activation.

In this study, we investigated the signaling pathway of the chemokine CCL18 on the lung cancer cell line A549 and on the breast cancer cell lines MDA-MB-231 and MCF-7. Breast cancer cell lines have been used because CCL18 has been best studied in breast cancer (17). Furthermore, a better understanding of CCL18 and its functions may lead to possible new treatment options in lung and breast cancer.

\section{Materials and Methods}

Cell culture. Before each experiment, the cells were cultured for a few days in the appropriate medium with $10 \%$ fetal calf serum (FCS) and $1 \%$ penicillin-streptomycin (P/S). Cells were cultured with phenol red-free medium, since phenol red may influence the binding of CCL18 to the estrogen affine receptors to be investigated due to its estrogen like structure. In some of the well plates containing A549 or MCF-7, $10 \mu \mathrm{l}$ of DMSO control or $10 \mu \mathrm{l}$ of 1 $\mathrm{mM} \mathrm{G}-15$ were added. The cell number was determined with an improved Neubauer hemocytometer.

Stimulation of cells. As we wanted to investigate the effect of CCL18 on GPR30 we needed to stimulate the cells with CCL18 in a well plate. A 1:10 dilution series was prepared in which either $10 \mu \mathrm{g} / \mathrm{ml}$,
$1 \mu \mathrm{g} / \mathrm{ml}, 100 \mathrm{ng} / \mathrm{ml}$ or $10 \mathrm{ng} / \mathrm{ml} \mathrm{CCL18} \mathrm{were} \mathrm{pipetted} \mathrm{into} \mathrm{each}$ reaction vessel, with final concentrations of $0.1,1,10$ and $100 \mathrm{ng} / \mathrm{ml}$ of CCL18 in the corresponding wells.

G15 and DMSO controls. In some experiments we inhibited GPR30 with G-15, which is an antagonist to GPR30 and which binds to GPR30 with high affinity. Therefore, in some experiments we inhibited GPR30 with G-1510 min before stimulation with CCL18 (100 $\mu \mathrm{M}$ final concentration of $\mathrm{G}-15$ for $\mathrm{MCF} 7$, and $10 \mu \mathrm{M}$ final concentration for A549). G-15 was dissolved in DMSO, a commonly used aprotic solvent. Since DMSO might have been able to stimulate the cells, DMSO was added to control wells. Subsequently, the cells were incubated for 24 hours in an incubator.

Harvesting cells with Trizol ${ }^{\circledR}$. In order to harvest the cells after stimulation with Trizol ${ }^{\circledR}$ Reagent, the supernatant of the wells was removed after 24 hours of incubation and stored at $-80^{\circ} \mathrm{C}$ for eventual ELISAs. $200 \mu \mathrm{l}$ of Trizol ${ }^{\circledR}$ were then pipetted into the wells. After a 5-min incubation, the cell components dissolved in Trizol ${ }^{\circledR}$ were filled into previously labeled reaction vessels and stored at $-80^{\circ} \mathrm{C}$.

Instructions from the Direct-zol ${ }^{\mathrm{TM}}$ RNA MiniPrep Instruction Manual were followed and isolation of the RNA, including DNAse I digestion, was performed to purify MALAT-1 from the genomic DNA.

Transcription. The RNA concentration was determined using the NanoDrop 2000c spectrophotometer and the associated software. The mRNA was transcribed into complementary DNA (cDNA) in order to use it for a PCR. For this purpose, $4 \mu \mathrm{l}$ of $5 \times$ iScript $^{\mathrm{TM}}$ reaction mix, $1 \mu 1$ of iScript ${ }^{\mathrm{TM}}$ reverse transcriptase and $15 \mu \mathrm{l}$ of diluted RNA were briefly spun down from the iScript ${ }^{\mathrm{TM}}$ cDNA Synthesis Kit and incubated in the thermocycler according to the iScript ${ }^{\mathrm{TM}}$ protocol.

Quantitative real-time polymerase chain reaction ( $q R T-P C R)$. The relative expression of MALAT-1 and the expression of MMP7 in relation to the expression of GAPDH were determined by means of quantitative real-time PCR. MCF-7 and MDA-MB-231 cells were used. Nutrient medium was used with FCS, with estrogen-free (charcoalfiltered) FCS, and without FCS, since FCS contains hormones and growth factors that can affect cell stimulation with CCL18.

After the mRNA has been transcribed into cDNA, the amount of double-stranded DNA was determined using SYBR-Green in the quantitative real-time PCR. Here, the property of SYBRGreen was exploited to fluoresce upon intercalation with double-stranded DNA. Accordingly, the degree of fluorescence correlates with the amount of double-stranded DNA and can be measured photometrically by the real-time PCR device. The Ct values of MALAT-1 and MMP7 were compared here with the $\mathrm{Ct}$ values of glyceraldehyde phosphate dehydrogenase (GAPDH).

The relative expression $(\mathrm{rE})$ of the target gene was calculated with the delta-delta-Ct method ( $\Delta \Delta \mathrm{Ct}$ method): $\mathrm{rE}=2 \mathrm{Ct}(\mathrm{GAPDH})$ -Ct (target gene) $\times 10,000$. Whether the inhibition of the GPR30 receptor with G-15 causes a difference in $\mathrm{rE}$ of MMP7 indirectly stimulated by CCL18 was also investigated. In addition, the relative expression of MMP14 was investigated, but below the detection limit (data not shown).

The following primers were used for PCR, based on Amplif ${ }^{\odot}$ library and synthesized by Biomers, Ulm, Germany: MMP7_1_F, MMP7_1_R, MMP7_1_forw, GAPdH_F, GAPdH_R, MALAT1_ hs_F1, MALAT1_hs_R1. 
Quantitative detection of EGF concentrations in the supernatants of the CCL18 stimulation series by EGF ELISA. EGF ELISA was performed according to the manufacturer's protocol of DuoSet ${ }^{\circledR}$ ELISA DEVELOPMENT SYSTEM (human EGF Catalog Number: DY236) (data not shown).

Investigation of ERK1/2 phosphorylation with In-Cell Western ${ }^{\circledR}$ s. LI-COR ${ }^{\circledR}$ Biosciences Protocol. In-Cell Western is an immunocytochemical assay designed to detect and quantify target structures in the cytosol of fixed cells via specific primary antibodies. Experiments were performed strictly according to LICOR ${ }^{\circledR}$ Biosciences Protocol.

Western blotting. Similar to the In-Cell Western ${ }^{\circledR}$ assay a western blot was performed. The following primers were used: MMP7_1_F, MMPz_1_R, MMP7_1_forw, GAPdH_F, GAPdH_R, MALAT1_hs_F1 and MALAT_1hs_R1. The following primary antibodies were used: ERK1/2 monoclonal mouse antibody (MK1):sc-13590 1:500 by Santa Cruz Biotechnology, Inc., Santa Cruz County, California, USA, p44/42 MAPK (Erk1/2) (3A7) monoclonal mouse antibody 1:1000 and phospho-p44/42 MAPK (Erk1/2) (Thr202/Tyr204) (D13.14.4E) XP monoclonal rabbit antibody 1:2000 each by Cell Signaling Technology, Danvers (MA, USA) and Boston, MA, USA.

The following secondary antibodies were used: IRDye $800 \mathrm{CW}$ donkey anti-rabbit 1:10000-1:40000 type IgG (H+L) [ID (P/N 92632213)] and IRDye 680LT donkey anti-mouse 1:20000-1:40000 IgG $(\mathrm{H}+\mathrm{L})$ [ID (P/N 926-68022)] each by LI-COR Biosciences in Lincoln, NE, USA.

Additives. Ham's F-12 (Caisson Labs, USA), DMEM (Gibco Invitrogen, Paisley, UK), FCS and estrogen-free FCS (GE Healthcare Bio-Science Austria GmbH, Pasching, Austria), penicillin, streptomycin and freezing serum with $20 \%$ DMSO and $80 \%$ FCS (Sigma-Aldrich, St. Louis, MO, USA).

All results are expressed as mean values with standard deviation.

Statistical analysis. All measured values are shown as mean value with standard deviation. RNA concentrations were determined using the NanoDrop 2000c spectrophotometer and the associated software. For evaluation of In-Cell Western ${ }^{\circledR}$ and western blotting, the plates and blots were analyzed with the Odyssey Infrared Imager and the Odyssey Infrared Imaging System Application Software Version 3.0 by LI-COR ${ }^{\circledR}$ Biosciences. The statistical significance of differences between the values of the controls and treated samples was determined by ANOVA using GraphPad Prism 8 and Excel. In each case, $* p<0.05$ and $* * p<0.01$ were considered statistically significant. ANOVA results are presented as $[\mathrm{F}$ (degrees of freedom between treatment group, degrees of freedom within treatment group) $=$ F statistic, $p$-value]. Tukey post - hoc tests were performed on statistically significant ANOVAs to determine which of the treatment groups were significantly different from each other.

\section{Results}

Determination of relative expression of MALAT-1 under the influence of CCL18. Very few studies on the signaling pathway of CCL18 have been performed so far. CCL18 induces epithelial-to-mesenchymal transition (EMT) in A549 (7) and metastasis associated lung adenocarcinoma transcript 1 (MALAT-1) expression is associated with EMT (18).
Assuming, that the long non-coding RNA (lncRNA) MALAT1 and CCL18 are both associated with carcinogenesis, we examined the expression of MALAT-1 in cancer cells previously stimulated with CCL18. MCF-7 showed a relatively high MALAT-1 expression, unstimulated and without fetal calf serum (FCS) or with estrogen-free FCS. A trend was observed where MALAT-1 expression decreases with increasing concentration of CCL18. In FCS-containing nutrient medium, low MALAT-1 expression was detected. In contrast, the relative expression of MALAT-1 in MDA-MB-231 revealed no evidence of dependence on the CCL18 concentration ( $p=0.997$, $\mathrm{F}=0.04$, critical $\mathrm{F}$ value=3.056). There was a non-significant tendency $(p=0.335, \mathrm{~F}=1.178$, critical $\mathrm{F}$ value=3.682) for the expression of MALAT-1 to be higher in cell culture medium with estrogen-free FCS and without FCS, as compared to medium containing regular FCS (data not shown).

Relative MMP7 expression (normalized to GAPDH) after CCL18 stimulation. Based on evidence for the possible involvement of matrix metalloproteinases (MMPs) in the transactivation of the EGF receptor via GPR30, the relative expression of matrix metalloproteinase 7 (MMP7) and matrix metalloproteinase 14 (MMP14) compared to GAPDH expression as a reference gene was investigated. In Figure 1, ANOVA showed that the relative expression of matrix metalloproteinase 7 in A549 cells was significantly affected by the nutrient medium, when treated with G-15, most likely due to estrogen like effects of the medium $[\mathrm{F}(2,36)=3.514$, $* p=0.040]$ and without $\mathrm{G}-15[\mathrm{~F}(2,30)=5.426, * * p=0.010]$. However, Tukey post-hoc tests revealed no significant difference between the different samples. Analogous to MALAT-1 expression, the relative expression of matrix metalloproteinase 7 (MMP7) was slightly higher in estrogenfree and FCS-free culture medium than in FCS-containing culture medium. Without FCS and with estrogen-free FCS in the cell culture medium relative MMP7 expression was strongest, in comparison to A549 with normal FCS. With increasing CCL18 concentration, relative MMP7 expression appeared to be decreasing, but not significantly. In FCScontaining medium, MMP7 expression in all wells, including controls, was approximately the same, regardless of CCL18 concentration. As shown in Figure 1, A549 cells were also treated with G-15 before stimulation with CCL18, in order to inhibit GPR30.

MMP7 expression also changed, depending on the medium, either with normal FCS, estrogen-free FCS or without FCS, where the relative MMP7 expression was most distinct. Analogous to the investigations of MALAT-1 expression, MMP7 expression was the strongest with estrogen-free FCS and barely detectable in cell culture medium with FCS. After inhibition with G-15, relative MMP7 expression was most distinct in medium with estrogen free FCS and without FCS. 
A



B

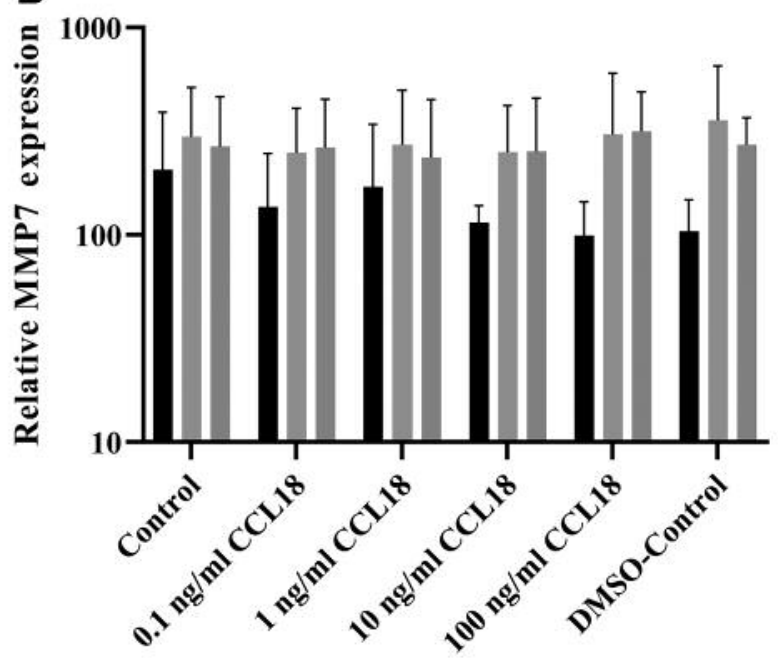

$10 \% \mathrm{FCS}$

Without Estrogen

Without FCS

Figure 1. Relative expression of matrix metalloproteinase 7 (MMP7) in relation to GAPDH expression in A549: Different media were used before and during stimulation. A549 were incubated $24 \mathrm{~h}$ before stimulation in their respective nutrient medium. Medium was used with 10\% FCS, with estrogen-free FCS and without FCS. From left to right, no stimulation (control), stimulated with $0.1 \mathrm{ng} / \mathrm{ml}, 1 \mathrm{ng} / \mathrm{ml}$, $10 \mathrm{ng} / \mathrm{ml}$ and $100 \mathrm{ng} / \mathrm{ml} \mathrm{CCL18} \mathrm{for} \mathrm{further} 24 \mathrm{~h}$. In (B), all cells except the DMSO control were treated with $10 \mu M G 1510$ min prior to stimulation. Simultaneously, the DMSO concentration corresponding to $10 \mu M$ G15 DMSO concentration was added to the control. The error bars represent the standard deviations [for (A) $n=3$ and $(B) n=3]$.
A

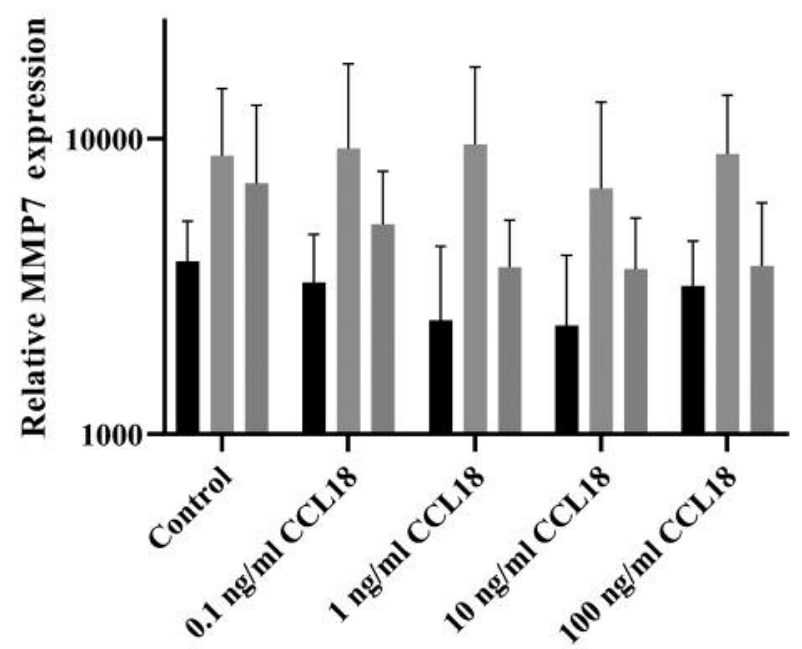

B



Figure 2. Relative expression of matrix metalloproteinase 7 (MMP7) in relation to expression of GAPDH in MCF-7: Before stimulation different media were used. MCF-7 were incubated $24 \mathrm{~h}$ before stimulation in their respective nutrient medium (see 4.1.8). Medium was used with $10 \%$ FCS, with estrogen-free FCS and without FCS. From left to right, cells either remained unstimulated (control) or were stimulated with $0.1 \mathrm{ng} / \mathrm{ml}, 1 \mathrm{ng} / \mathrm{ml}, 10 \mathrm{ng} / \mathrm{ml}$ and $100 \mathrm{ng} / \mathrm{ml} \mathrm{CCL18} \mathrm{for} \mathrm{a} \mathrm{further} 24 \mathrm{~h} . \mathrm{In}$ (B), cells were treated with $10 \mu \mathrm{M}$ G15 $10 \mathrm{~min}$ prior to stimulation, except for the DMSO control. Tukey post-hoc tests revealed significant differences with estrogen-free FCS and G-15 between the control and 1

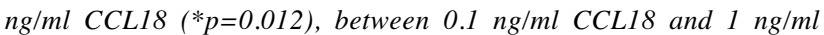
CCL18 (*p=0.011) and between $1 \mathrm{ng} / \mathrm{ml} \mathrm{CCL18}$ and $10 \mathrm{ng} / \mathrm{ml} \mathrm{CCL18}$ $\left({ }^{*} p=0.037\right)$ [mean value $\pm S D$, for $(A)$ and $(B), n=3$, respectively]; $* p<0.05$. 
Without G-15 inhibition, it was possible to show a dependence of the expression on the CCL18 concentration. With increasing CCL18 concentration, MMP7 expression increased not significantly $[\mathrm{F}(4,30)=1.391, p=0.261]$. As a control, in the stimulation series with G15, the A549 were treated in a well with $100 \mu \mathrm{M}$ DMSO because DMSO was used as a lipophilic solvent for G15. There were no significant differences between the DMSO-containing and the DMSO-free control. However, MMP7 expression was slightly lower in the DMSO-containing medium with $10 \%$ FCS than in the DMSO-free control.

The relative expression of MMP7 in MCF-7 cells shown in Figure 2 also showed a dependence on the cell culture medium used, utilizing two-way $\operatorname{ANOVA}[\mathrm{F}(2,30)=6.136$, $* * p=0.006]$ without $\mathrm{G} 15$ inhibition and $[\mathrm{F}(2,36)=7.370$, $* * p=0.002]$ with G15. However, expression was strongest under the influence of "estrogen-free" FCS. FCS-free culture medium had the second highest relative MMP7 expression normalized to GAPDH and medium with $10 \%$ FCS the lowest expression. In the stimulation series with G15, however, this ratio already changed in the DMSO control in favor of the FCS-free medium with the strongest MMP7 expression and in descending order the medium with estrogen-free FCS and with the lowest MMP7 expression with $10 \%$ FCS in the medium.

As with A549, in the stimulation series without G15, MCF7 cells without FCS also showed decreasing MMP7 expression by up to $50 \%$ between the control and the cells treated with $10 \mathrm{ng} / \mathrm{ml} \mathrm{CCL18,} \mathrm{depending} \mathrm{on} \mathrm{increasing}$ CCL18 concentration. At $100 \mathrm{ng} / \mathrm{ml}$ of CCL18, MMP7 expression tended to increase slightly, regardless of the nutrient medium. Figure 2 shows the relative expression of matrix metalloproteinase 7 in MCF-7 cells, under GPR30 inhibition with G-15, analogous to Figure 1 for A549. Noticeable was the generally low relative expression, which, compared to the A549, was even more noticeable here.

Again, the dependence of MMP7 expression on the estrogen content of the culture medium was shown $[\mathrm{F}(2$, $30)=6.136, * * p=0.006]$. Analogous to the study of relative MALAT-1 expression, MMP7 expression without FCS and with estrogen-free FCS was the strongest and hardly detectable in FCS-containing nutrient medium, independent of the CCL18 concentration $[\mathrm{F}(4,30)=0.324, p=0.860]$. A dependence of MMP7 expression on the CCL18 concentration could not be detected in FCS-containing nutrient medium. However, as in the case of the A549, the inhibition of GPR30 with G15 seemed to reverse the dependence of MMP7 expression on the nutrient medium $[\mathrm{F}(2,36)=7.370, * * p=0.002]$. Tukey post -hoc tests revealed significant differences with estrogen-free FCS and G-15 between the control and $1 \mathrm{ng} / \mathrm{ml} \mathrm{CCL18}\left({ }^{*} p=0.012\right)$, between $0.1 \mathrm{ng} / \mathrm{ml} \mathrm{CCL} 18$ and $1 \mathrm{ng} / \mathrm{ml} \mathrm{CCL18}(* p=0.011)$ and between $1 \mathrm{ng} / \mathrm{ml} \mathrm{CCL18}$ and $10 \mathrm{ng} / \mathrm{ml} \mathrm{CCL18} \mathrm{(*p=0.037).}$
We observed that relative expression of MMP7 in MCF-7 cells was very low compared to the relative expression of MMP7 at the non-G-15 treated MCF-7, after addition of G1510 min prior to stimulation with CCL18, regardless of the CCL18 concentration (mean value of controls with G$15=22.00, \mathrm{SD}=4.56$ versus a mean value of the controls without $\mathrm{G}-15=6552.41, \mathrm{SD}=4508.39, * * p<0.001)$.

MMP14 expression in A549 was extremely low in qPCR, which is why MMP14 has not been further investigated (results not shown).

EGF-ELISA after stimulation with CCL18. Although the EGF ELISA performed twice with the cell culture supernatants from the experiments with MCF7 and A549 after CCL18 stimulation gave an adequate standard curve, the measured values were all in the range of zero or less than the range measurable for the device. EGF could not be detected in the unstimulated cell culture supernatants neither in A549 nor in MCF7 cells. Also, upon stimulation with CCL18 at any of the concentrations used, none of the cell lines tested here could induce the release of EGF.

ERK phosphorylation via CCL18 in MCF-7. Here, the change of the ratio of phosphorylated ERK and non-phosphorylated ERK under the influence of different concentrations of CCL18, estrogen (estradiol) and EGF was examined at the intracellular level. Phosphorylated ERK is shown in red and non-phosphorylated in green (Figure 3). The wells, whose cells had separated during the washings, were not included in the evaluation. The normalized mean fluorescence intensities are described in Figure 4. One-way ANOVA tests on MCF-7 cells revealed a significant difference between the treatments $[\mathrm{F}(13,126)=7.055, * * p<0.0001]$. Tukey post-hoc tests revealed a significant increase in ERK phosphorylation with EGF $5 \mathrm{ng}(* p=0.035)$ and EGF $10 \mathrm{ng}(* * p<0.0001)$, each compared to the control group.

ERK phosphorylation via CCL18 in A549. Unstimulated A549 are significantly more phosphorylated than unstimulated MCF-7. The cells with the lowest ERK phosphorylation rate are those inhibited with G-15 and stimulated with CCL18. With G15, ERK activation is lower with all CCL18 concentrations compared to the control, except with $1 \mathrm{ng} / \mathrm{ml}$ CCL18. All other stimulus series appear orange with greater phosphorylation or black when the cells have detached from the wells. The details of the stimulation series are described in Figure 5.

A549 were significantly more sensitive in the washes than the MCF-7, as they were easier to break out of the wells and the study was thus partially lost. The wells from which cells have dissolved more frequently show a greater standard deviation in summary than those from which the cells did not dissolve. 


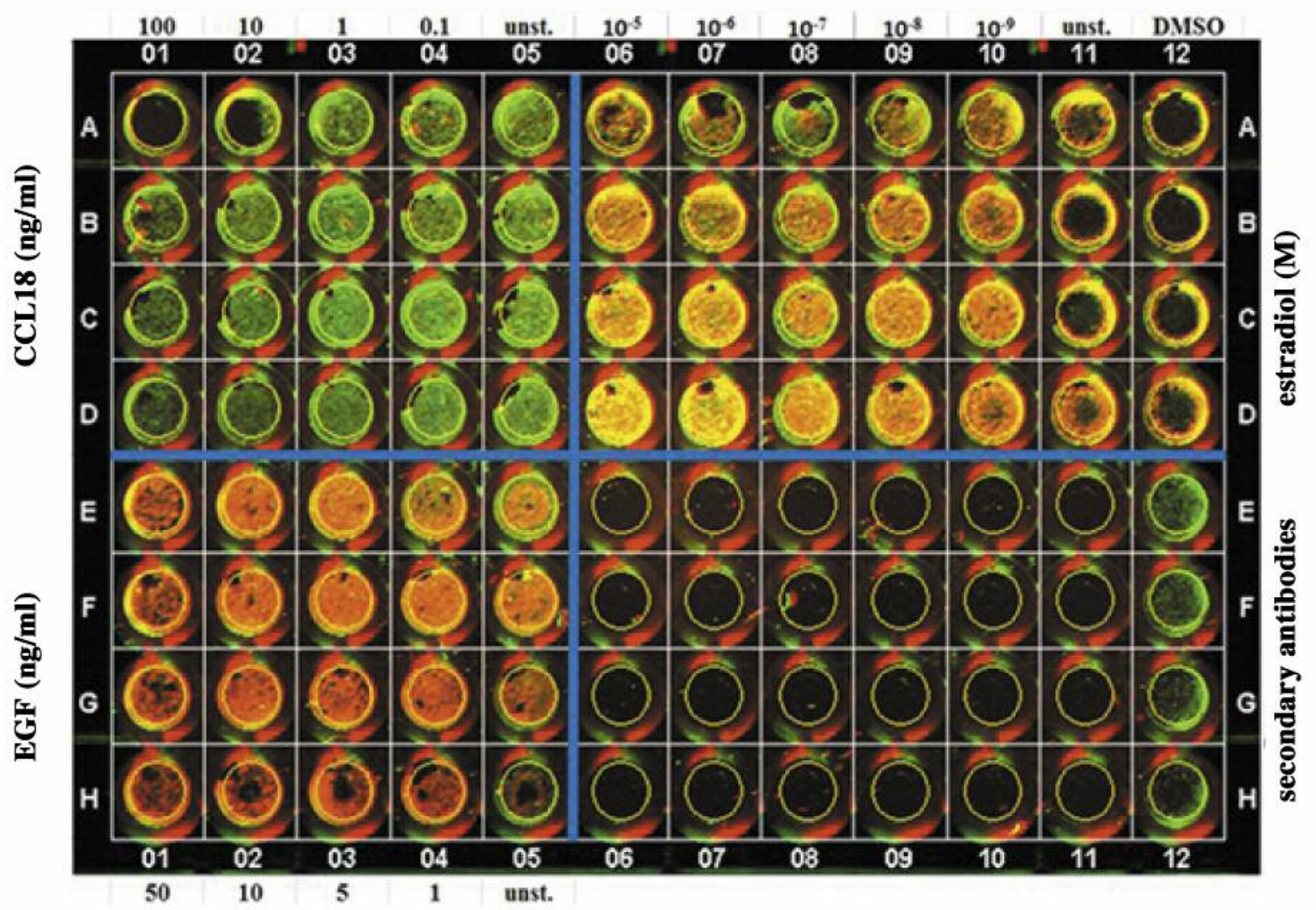

Figure 3. In-Cell Western ${ }^{\circledR}$ extracellular signal-regulated kinase (ERK) phosphorylation scan with MCF-7: Stimulation series with CCL18: Unstimulated: Row 05A to D; $0.1 \mathrm{ng} / \mathrm{ml}$ CCL18: Row 04A to D; $1 \mathrm{ng} / \mathrm{ml} \mathrm{CCL18:} \mathrm{Row} \mathrm{03A} \mathrm{to} \mathrm{D;} 10 \mathrm{ng} / \mathrm{ml} \mathrm{CCL18:} \mathrm{Row} \mathrm{02A} \mathrm{to} \mathrm{D;} 100 \mathrm{ng} / \mathrm{ml}$ CCL18: Row 01A to D; Stimulation series with estradiol: DMSO control: 12A to D; unstimulated: Row 11A to D; 10-9 mol/l estradiol: Row 10A to D; 10-8 mol/l estradiol: Row 09A to D; 10-7 mol/l estradiol: Row 08A to D; 10-6 mol/l estradiol: Row 07A to D; 10-5 mol/l estradiol: Row 06A to D; Stimulation series with EGF: unstimulated: Row 05E to H; $1 \mathrm{ng} / \mathrm{ml}$ EGF: Row 04E to H; $5 \mathrm{ng} / \mathrm{ml}$ EGF: Row 03E to H; $10 \mathrm{ng} / \mathrm{ml}$ EGF: Row $02 \mathrm{E}$ to $\mathrm{H} ; 100 \mathrm{ng} / \mathrm{ml}$ EGF: Row $01 \mathrm{E}$ to $\mathrm{H}$; Control with secondary antibodies without primary antibodies: Row $12 \mathrm{E}$ to $\mathrm{H}$.

For ERK phosphorylation in A549, one-way ANOVA showed significant difference among the means $[\mathrm{F}(19$, 180) $=2.078, * * p=0.007]$.

Only after adding G15, the ERK phosphorylation is attenuated slightly, but not significantly ( $p>0.05$ for each sample compared to the controls). The degree of phosphorylation tended to increase with increasing concentration. Under estradiol, the level of phosphorylated ERK at each concentration was slightly above the level of phosphorylated ERK in the control. As with MCF7, the proportion of phosphorylated ERK tended to increase with increasing levels of EGF. The increase, however, peaked at 50 $\mathrm{ng} / \mathrm{ml}$ EGF, with a sharp drop at $10 \mathrm{ng} / \mathrm{ml}$, which can be attributed to the cells released from the wells. The A549 treated previously and under CCL18 stimulation with G15 showed no change in the level of phosphorylated ERK compared to the control (Figure 5).

ERK phosphorylation after CCL18 stimulation coincubated with indomethacin, SQ 22536 and dbcAMP. MCF-7 showed no significantly $(p>0.05)$ lower ERK phosphorylation than the unstimulated cells using the adenylate cyclase inhibitor SQ 22536 or the nonselective cyclooxygenase inhibitor indomethacin. By combining the two inhibitors with $10 \mathrm{ng} / \mathrm{ml}$ CCL18, only with indomethacin a slight but not significant increase in phosphorylation was shown in ICW (data not shown).

After the A549 had been stimulated with dbcAMP, there was an increase in the ERK-phosphorylation. ERK phosphorylation after CCL18 stimulation was also increased in this experiment compared to the control. The A549 showed a higher proportion of phosphorylated ERK after incubation with dbcAMP, but not as high as in pure CCL18 stimulation. With additional stimulation of dbcAMP pretreated cells with CCL18, the proportion of phosphorylated ERK did not increase more than under dbcAMP inhibition alone (data not shown).

Examination of results from the ICW by repeating with western blot: ERK Phosphorylation at A549 under CCL18, 


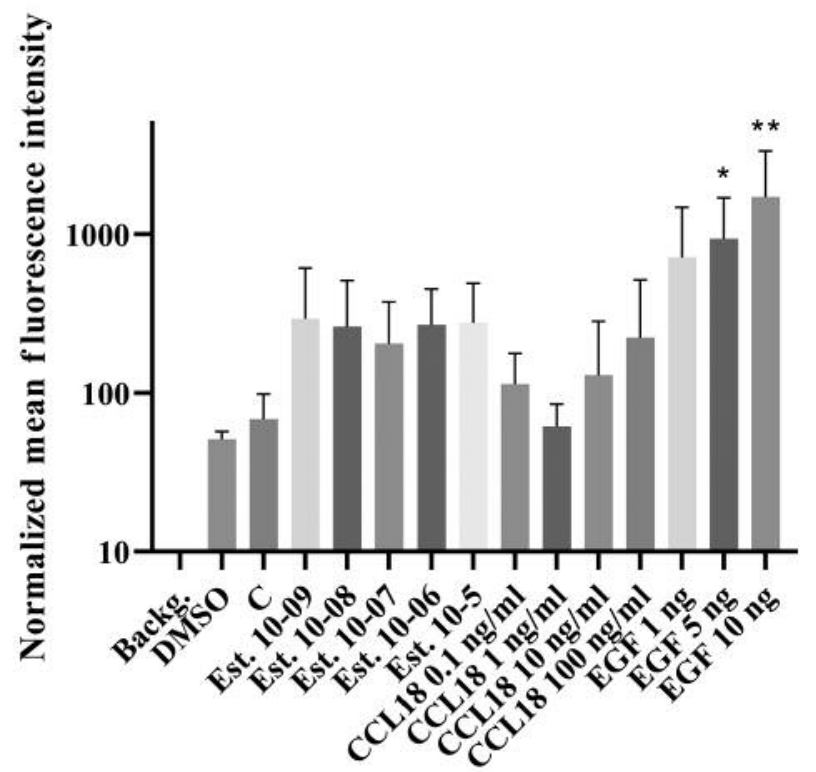

Figure 4. In-Cell Western ${ }^{\circledR}$ extracellular signal-regulated kinase (ERK) phosphorylation in MCF-7: MCF-7 was stimulated for 10 minutes with CCL18, estradiol and EGF at different concentrations, unstimulated (control), or incubated with DMSO as a control. Subsequently, the cells were treated according to the protocol of LI-COR ${ }^{\circledR}$ Biosciences. phosphorylated ERK is shown in red, non-phosphorylated ERK in green (mean value $\pm S D 4$ wells each, $n=3$ ); $* p<0.05$, $* * p<0.001$.

with $d b c A M P$. ERK phosphorylation after CCL18 stimulation versus control was slightly but not significantly increased. A549 cells showed, after incubation with dbcAMP, a lower proportion of phosphorylated ERK compared to the control. With additional stimulation of dbcAMP-pretreated cells with CCL18, the proportion of phosphorylated ERK did not increase more than under dbcAMP inhibition alone (Figure 6).

\section{Discussion}

CCL18 influences numerous tumor diseases, inflammatory and allergic diseases, including the lung, skin and joints, as a very complex signaling protein (7). In NSCLC, liver, breast and adenocarcinoma of the pancreas, CCL18 promotes epithelialto-mesenchymal transition (EMT) and thus metastasis of cancer cells $(8,9)$. Increased serum CCL18 levels in patients with adenocarcinoma of the lungs correlate with a shortened overall survival and tissue infiltration of CCL18-expressing tumor-associated macrophages (TAM) in patients with breast cancer is an unfavorable prognostic marker and correlates with shortened overall survival $(6,12,19)$.

In contrast, CCL18 expression measured directly in gastric carcinoma tissue correlates with both prolonged overall survival and prolonged disease-free survival (20). In patients



Figure 5. In-Cell Western ${ }^{\circledR}$ extracellular signal-regulated kinase (ERK) phosphorylation in A549: A549 were stimulated for 10 minutes with CCL18, estradiol and EGF at different concentrations or unstimulated (C) or incubated with DMSO as control. Subsequently, the cells were treated according to the protocol of LI-COR ${ }^{\circledR}$ Biosciences. Those cells additionally receiving DMSO control were either treated with 10umol/l G-15 either 10 minutes prior to or during stimulation, or stimulated with estrogen dissolved in DMSO, see lettering (A). Red areas correspond to phosphorylated ERK, green areas correspond to non-phosphorylated ERK. The error bars represent the standard deviations $(n=3)$.

with acute lymphoblastic leukemia, elevated levels of CCL18 are found in the serum (3), whereas in patients with ovarian cancer, ascites is more common (21). In vitro, CCL18 profibrotically acts on fibroblasts via ERK activation. With regard to lung disease, elevated serum levels also occur in fibrosis-associated lung diseases such as sarcoidosis and idiopathic pulmonary fibrosis $(18,21,22)$. There is evidence that CCL18 inhibits airway responsiveness and inflammatory responses in the lung (21). These numerous properties make CCL18 a potential therapeutic target in diverse diseases.

Metastasis correlates with a poor prognosis in tumor patients. EMT plays a crucial role in the metastasis of tumors. Cancer precursors can, according to the current literature, undergo EMT at every stage of differentiation, depending on genetic factors and factors from the vicinity of the cancer cells (23-26). CCL18-stimulated A549 responded with EMT: the EMT-correlated transcription factor SNAI1 was induced, E-cadherin as an epithelial marker decreased, whereas the concentration of mesenchymal marker FSP1 increased (7). 




Figure 6. Western blot: Detection of phosphorylated and non-phosphorylated ERK 1 and 2 in A549: Green bands correspond to phosphorylated extracellular signal-regulated kinase (ERK), red bands correspond to non-phosphorylated ERK. The size marker is shown on the left. The A549 were previously stimulated according to the labeling $(n=3)$.

The lack of expression of the IncRNA MALAT-1 caused a decrease in the EMT-associated genes ZEB1, ZEB2 and the transcriptional repressor SNAI1 and an increase in Ecadherin expression in bladder carcinoma cells as well as a decrease in EMT-associated genes in A549 $(25,26)$. High MALAT-1 expression in NSCLC is associated with increased cell proliferation, invasive growth, and unfavorable prognosis (26). On the other hand, in the breast cancer cell lines MDA-MB-231 and MCF-7, a knockdown of lncRNA MALAT-1 by lentiviral RNA induced the induction of various EMT-associated markers such as cadherin 2 and $\mathrm{N}$ cadherin, whereas epithelial markers such as cadherin 1 and E-cadherin were down regulated. In breast cancer cells, therefore, metastasis appears to be favored by decreased
MALAT-1 expression $(19,25,26)$. MALAT- 1 and CCL18 are both involved in EMT. Therefore, the relationship of CCL18-stimulated MCF-7 and MDA-MB231 cell lines was investigated in this work.

In terms of the objective of this work, quantitative realtime PCR analysis revealed differential results for each breast cancer cell line. Both CCL18 and MALAT-1 are involved in EMT. The literature on the investigated breast cancer cell lines MCF-7 and MDA-MB-231 seems to favor both low MALAT-1 expression and high CCL18 expression via TAM EMT and metastasis $(19,25,27)$. It was therefore necessary to clarify whether CCL18 has an influence on MALAT-1 expression. It was found that the relative expression of MALAT-1 under stimulation with CCL18 was 
not significantly affected in MCF-7 and MDA-MB-231 cells. In MCF-7, there was a non-significant tendency to see relative expression decrease with increasing concentration of CCL18. It can be assumed that the different expression of CCL18 affinity surface receptors of the examined cell lines is responsible for the slightly different results. Filardo et al. were able to show that estrogen activates GPR30 (14). Since estrogen and CCL18 both activate GPR30 as a common receptor, they hypothesized that estrogen and CCL18 influence expression of MALAT-1 via the same or a similar pathway and relative MALAT-1 expression should behave similarly to increasing CCL18 concentration.

The present results support this hypothesis of a common signaling pathway of CCL18 and estrogen, since MDA-MB231 hardly express $\operatorname{ER} \alpha$ and $\operatorname{ER} \beta(17,28)$. In contrast, MDA-MB-231 express CCL18-affine PITPNM3 and GPER receptors $(28,29)$. The relative MALAT-1 expression is already very low in the control there and barely changes with increasing CCL18 concentration, independently of the estrogen content of the nutrient medium of the cells. Therefore, MALAT expression of MDA-MB-231 and MCF7 under estrogen stimulation does not appear to be solely from the receptor GPER shared with CCL18, but rather, e.g. in MCF7 depend on the other estrogen receptors, including $\mathrm{ER} \alpha$ and ER $\beta$. Since MCF-7, in contrast to MDA-MB-231, express $E R \alpha$ and $E R \beta$ receptors in addition to GPR30, a concentration-dependent change in MALAT-1 expression is to be expected here. The lower the estrogen content of the nutrient medium and the higher the CCL1 8 concentration, the lower the relative MALAT-1 expression. Therefore, the presence of several different signaling pathways for CCL18 and estrogen seems to be responsible for the opposite reaction of relative MALAT-1 expression. In addition, these experiments have also been carried out with RLE-6TN, a rat cell line of the alveolar epithelial type II cells. In the experiments performed with RLE-6TN there could be observed a slight but not significant tendency for a stronger MALAT-1 expression with FCS than without FCS or in estrogen free FCS, but no clear correlation with the CCL18 concentration. The experiments with this cell line are not further discussed here.

It has been shown that the chemokine CCL18 can activate the GPR30 receptor (3). Filardo et al. showed that the estrogen estradiol activates the GPR30 receptor and, in contrast to the estrogen receptors $\operatorname{ER} \alpha$ and $\operatorname{ER} \beta$, acts via a fast pathway via cAMP and the ERK signaling pathway. GPR30 activates adenylate cyclase via the Gas subunit and thus increases the intracellular cAMP concentration, which in turn activates further signaling pathways. GPR30 activation also leads to the activation of matrix metalloproteinases via the Src-like kinase-dependent G $\beta \gamma$ subunit, which in turn causes cleavage of cell membrane-bound heparin-binding EGF and via $\beta$-arrestin to other signaling pathways, including the oncogenic Src, Akt and ERK signaling pathways. This will activate the ERK signaling pathway via the EGF receptor $(13,15,16)$. The individual sections of this pathway were investigated in this work.

Instead of estrogen as in the work by Filardo et al., this time the stimulated GPR30 with CCL18 was based on the hypothesis that if CCL18, as well as estrogen activates GPR30, the signaling pathway would have to be the same. Due to the strong association of CCL18 overexpression and NSCLC lung cancer and breast cancer, A549 (alveolar adenocarcinoma of the lung) and MCF-7 and MDA-MB231 cells (breast cancer cell lines) were studied. Again, qPCR gave different results for each cell line. There was a significant tendency that the relative expression of matrix metalloproteinase 7 in A549 is dependent on the estrogen content of the nutrient medium. The nutrient medium used in A549 was DMEM. Stimulation series were performed with $10 \%$ FCS (high estrogen-concentration), with $10 \%$ estrogen "free" FCS (with little estrogen) and without FCS (no estrogen). Under stimulation with all CCL18 concentrations, a clear difference in expression between the estrogen content of the nutrient medium was detected, when GPR30 was inhibited by G-15 $[\mathrm{F}(2,36)=3.514, * p=0.040]$.

Without FCS, MMP7 expression was the strongest in the A549 and the second most potent with estrogen-free FCS. As the concentration of CCL18 increased, MMP7 expression appeared to decrease non significantly under estrogen-free nutrient medium; estrogen-free FCS and classical FCS appeared to suppress MMP7 expression from the outset even in the control. Estrogen "free" FCS has been filtered through charcoal and still contains 10 to $20 \mathrm{pg} / \mathrm{ml}$ of estrogen according to the manufacturer's instructions. In contrast, classical FCS contains about 33 to $35 \mathrm{pg} / \mathrm{ml}$ estrogen. Assuming, that the estrogen "free" FCS still has a pronounced estrogenic activity, estrogen appears to inhibit MMP7 expression. In FCS-free and consequently estrogenfree culture medium CCL18 takes over the role of inhibiting MMP7, but only from higher concentrations.

Inhibition of GPR30 with G15 before and during stimulation with CCL18 described above resulted in significantly lower relative expression of MMP7 in MCF-7 cells, between the control groups with and without G15, regardless of the concentration of CCL18 $(p<0.01)$. On the other hand, if estrogen inhibits MMP7 expression via activation of GPR30, an increase in MMP-7 expression would have been expected under GPR30 inhibition with G15, regardless of CCL18 concentration or estrogen concentration.

qPCR with MMP14 in A549 cells resulted in a maximum relative expression of 20 (relative to GAPDH expression), which is relatively low. Therefore, MMP14 has not been further investigated (results not shown). The results suggest that GPR30 plays a role in the induction of matrix metalloproteinase 7 expression in A549. However, this result 
cannot be reconciled with the signal pathway from Filardo et al. since the relative expression of MMP7 is inhibited by rising estrogen concentrations stronger than by increasing CCL18 concentrations.

Internal laboratory FACS analyzes have shown that MCF7 express GPR30 significantly more than A549. Comparing the effect of different media on MMP7 expression, the relative expression of MMP7 (to GAPDH expression) in MCF-7 with estrogen-free FCS is approximately half that of medium containing estrogen-containing FCS. When inhibited with G15, the relative expression ( $\mathrm{rE}$ ) remained consistently low

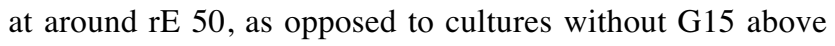
$\mathrm{rE} \mathrm{1000.} \mathrm{The} \mathrm{difference} \mathrm{in} \mathrm{CCL18} \mathrm{causes} \mathrm{little} \mathrm{difference} \mathrm{in}$ MMP7 expression. Thus, without GPR30, MCF-7 does not appear to be able to express MMP7.

The different response to the estrogen content of the nutrient medium of MMP7 expression in MCF7 compared to that of A549 can be explained most easily by the different receptor expression. According to laboratory internal investigations, MCF7 express ER $\alpha$, ER $\beta$ and more GPR30 than A549. Differentially expressed receptor expressions could be responsible for this estrogen dependent MMP7 expression. Under isolated consideration of the signaling pathway via GPR30, the MCF7 cells would have had to react more strongly to different CCL18 concentrations because of the higher GPR30 expression compared to A549.

This suggests that GPR30 plays a major role in inhibiting the expression of matrix metalloproteinases in MCF7. Inhibition of the GPR30 receptor with G15 in MCF7 significantly suppresses MMP7 expression, even more so than in A549. As with A549, there was no clear correlation between MMP7 expression and CCL18 concentration in MCF7 inhibited with G15.

Filardo et al. have described membrane-bound HB-EGF in the estradiol-activated GPR30 signaling pathway which is cleaved from the cell surface upon activation of GPR30 by matrix metalloproteinases $(14,15)$. Activation of the GPR30 with CCL18 would therefore also have to lead to a release of HB-EGF. On the other hand, the stimulation of A549 and MCF7 with CCL18 has in each case led to an inhibition of the expression of the supposedly HB-EGF releasing MMP7. Thus, the result without detection of released HB-EGF is consistent with the results of studies on MMP7 expression in A549 and MCF7.

FACS analyzes of the surface receptors revealed lower GPR30 expression in A549, whereas in MCF7 increased GPR30 expression was detected (data not shown). According to the results from the investigation of ERK phosphorylation with in-cell Western and western blotting, the stimulated Gas protein-coupled receptor GPR30 inhibits ERK phosphorylation via an increased intracellular cAMP concentration. Even the controls show that the ERK phosphorylation of the A549 is stronger than ERK phosphorylation in MCF7.
Due to the different expression on the cell surface in the A549, the signaling pathway via GPR30 and thus the ERK activation is less predominant. GPR30 is significantly less expressed in A549 than in MCF7. Signaling via GPR30 dominates and ERK activation is inhibited. Apparently, CCL18 causes a rapid signal transduction via these two Gprotein-coupled receptors, as has already been demonstrated by Filardo for the ligand estradiol at GPR30 (14). Thus, inhibition of GPR30 with G15 prior to stimulation with CCL18 would require even greater ERK activation. Curiously, however, even though it was not significant, ERK activation was diminished (Figure 5). This indicates that possibly other receptors might also play a role in the CCL18 signaling pathway. Estradiol caused ERK activation in MCF7 even in very low concentrations. Under stimulation with EGF ERK activation was stronger than under estradiol and showed a concentration-dependent course. In A549, ERK activation under stimulation with estradiol is as pronounced as ERK activation under EGF stimulation. This confirms the signaling pathway described by Filardo et al. (16).

Influence of indomethazine, dbcAMP and SQ22536 on stimulation of cells with CCL18. The result of ERK phosphorylation after stimulation with CCL18 in in-cell Western led to the question of how a reduction or increase of the intracellular cAMP level affects this hypothetical pathway. Inhibition of adenylate cyclase with SQ22536 or indirect inhibition with the cyclooxygenase inhibitor indomethazine and thus lowering of the intracellular cAMP level resulted in reduced ERK activation in MCF-7. This result would again be better with that of Filardo et al. known pathway for estrogen via the EGFR, since this leads to ERK activation independently of cAMP (14). If dbcAMP is present in high concentration intracellularly, ERK activation is inhibited. Addition of CCL18 will then cause no further ERK activation (data not shown). These results could be confirmed via western blot. In addition, the activation of ERK1 and ERK2 could be differentiated. The activation of ERK1 as a function of the dbcAMP and CCL18 concentrations was like the activation of ERK2 (data not shown). Also, Atamas et al. were able to detect ERK activation by recombinant human CCL18 in lung and dermal fibroblasts via western blot. In Atamas et al. however, ERK1 seems to be more phosphorylated than ERK2 $(30,31)$. It would also have been expected that direct inhibition of adenylate cyclase with SQ22536 or indirect inhibition with indomethazine via COX2 and thus PEG2 synthesis would result in ERK phosphorylation. Accordingly, in MCF7 SQ22536 or indomethazine led to fewer ERK activation, but surprisingly CCL18 stimulation in combination with SQ22536 or indomethazine led to high ERK activation. 
The estradiol signaling pathway described by Filardo et al. would have to be examined in more detail for CCL18. However, the interaction between estrogen and GPR30 and between CCL18 and GPR30 seem to be fundamentally different (32). Since stimulation of GPR30 with CCL18 leads to a decrease in MMP7 expression, it is unlikely that CCL18 can activate the signaling pathway described by Filardo via GPR30, MMP and EGFR. In this signaling pathway, cleavage of HB-EGF is dependent on MMP. Nevertheless, the experiment should be repeated under optimized conditions.

In addition to the investigated matrix metalloproteinases 7 and 14 , a determination of the relative expression of other matrix metalloproteinases after CCL18 stimulation would be desirable. In addition, the promiscuity typical of chemokines and chemokine receptors could be responsible for the signaling pathway after GPR30 activation of CCL18 being very different from the estrogen signaling pathway $(30,32)$. This means that the signaling pathways are different.

\section{Conflicts of Interest}

The Authors declare no conflicts of interest regarding this study.

\section{Authors' Contributions}

Roland G. W. Schmidt-Wolf designed and performed the experiments, analyzed the data and wrote the manuscript. Gernot Zissel conceived and designed the experiments, analyzed the data and corrected the paper.

\section{Acknowledgements}

The Authors thank Mr. PD Wolfgang Schäfer, Freiburg, for his support. In particular, they would like to thank Dr. Ing. Kerstin Höhne, Freiburg.

\section{References}

1 Robert Koch-Institut: Bericht zum Krebsgeschehen in Deutschland 2016: 274, 2016.

2 Guibert N, Ilie M, Léna H, Didier A, Hofman P and Mazieres J: KRAS and bronchial adenocarcinoma. Between disappointments and hopes. Rev Mal Respir 33(2): 156-164, 2016. PMID: 26520779. DOI: 10.1016/j.rmr.2015.05.012

3 Catusse J, Wollner S, Leick M, Schröttner P, Schraufstätter I and Burger M: Attenuation of CXCR4 responses by CCL18 in acute lymphocytic leukemia B cells. J Cell Physiol 225(3): 792-800, 2010. PMID: 20568229. DOI: 10.1002/jcp.22284

4 von Minckwitz G, Schwedler K, Schmidt M, Barinoff J, Mundhenke C, Cufer T, Maartense E, de Jongh FE, Baumann KH, Bischoff J, Harbeck N, Lück HJ, Maass N, Zielinski C, Andersson M, Stein RC, Nekljudova V and Loibl S: Trastuzumab beyond progression: overall survival analysis of the GBG 26/BIG 3-05 phase III study in HER2-positive breast cancer. Eur J Cancer Oxf Engl 1990 47(15): 2273-2281, 2011. PMID: 21741829. DOI: 10.1016/j.ejca.2011.06.021
5 Islami F, Torre LA and Jemal A: Global trends of lung cancer mortality and smoking prevalence. Transl Lung Cancer Res 4(4): 327-338, 2015. PMID: 26380174. DOI: 10.3978/j.issn.22186751

6 Arnold M, Karim-Kos HE, Coebergh JW, Byrnes G, Antilla A, Ferlay J, Renehan AG, Forman D and Soerjomataram I: Recent trends in incidence of five common cancers in 26 European countries since 1988: Analysis of the European Cancer Observatory. Eur J Cancer Oxf Engl 1990 51(9): 1164-1187, 2015. PMID: 24120180. DOI: 10.1016/j.ejca.2013.09.002

7 Ploenes T, Scholtes B, Krohn A, Burger M, Passlick B, MüllerQuernheim J and Zissel G: CC-chemokine ligand 18 induces epithelial to mesenchymal transition in lung cancer A549 cells and elevates the invasive potential. PloS One 8(1): e53068, 2013. PMID: 23349697. DOI: 10.1371/journal.pone.0053068

8 Schutyser E, Richmond A and Van Damme J: Involvement of CC chemokine ligand 18 (CCL18) in normal and pathological processes. J Leukoc Biol 78(1): 14-26, 2005. PMID: 15784687. DOI: $10.1189 / \mathrm{jlb} .1204712$

9 Nawshad A, Lagamba D, Polad A and Hay ED: Transforming growth factor-beta signaling during epithelial-mesenchymal transformation: implications for embryogenesis and tumor metstasis. Cells Tissues Organs 179(1-2): 11-23, 2005. PMID: 15942189. DOI: $10.1159 / 000084505$

10 Zhao C, Zheng S, Yan Z, Deng Z, Wang R and Zhang B: CCL18 promotes the invasion and metastasis of breast cancer through Annexin A2. Oncol Rep 43(2): 571-580, 2020. PMID: 31894281. DOI: $10.3892 /$ or.2019.7426

11 Hieshima K, Imai T, Baba M, Shoudai K, Ishizuka K, Nakagawa T, Tsuruta J, Takeya M, Sakaki Y, Takatsuki K, Miura R, Opdenakker G, Van Damme J, Yoshie O and Nomiyama H: A novel human $\mathrm{CC}$ chemokine PARC that is most homologous to macrophage-inflammatory protein-1 alpha/LD78 alpha and chemotactic for $\mathrm{T}$ lymphocytes, but not for monocytes. J Immunol 159(3): 1140-1149, 1997. PMID: 9233607.

12 Pandey DP, Lappano R, Albanito L, Madeo A, Maggiolini M and Picard D: Estrogenic GPR30 signalling induces proliferation and migration of breast cancer cells through CTGF. EMBO J 28(5): 523-532, 2009. PMID: 19153601. DOI: 10.1038/emboj.2008.304

13 Zlotnik A and Yoshie O: Chemokines: a new classification system and their role in immunity. Immunity 12(2): 121-127, 2000. PMID: 10714678. DOI: 10.1016/s1074-7613(00)80165-x

14 Filardo EJ, Quinn JA, Bland KI and Frackelton AR: Estrogeninduced activation of Erk-1 and Erk-2 requires the G proteincoupled receptor homolog, GPR30, and occurs via transactivation of the epidermal growth factor receptor through release of HB-EGF. Mol Endocrinol Baltim Md 14(10): 16491660, 2000. PMID: 11043579. DOI: 10.1210/mend.14.10.0532

15 Filardo EJ: Epidermal growth factor receptor (EGFR) transactivation by estrogen via the G-protein-coupled receptor, GPR30: a novel signaling pathway with potential significance for breast cancer. J Steroid Biochem Mol Biol 80(2): 231-238, 2002. PMID: 11897506. DOI: 10.1016/s0960-0760(01)00190-x

16 Filardo EJ and Thomas P: GPR30: a seven-transmembranespanning estrogen receptor that triggers EGF release. Trends Endocrinol Metab TEM 16(8): 362-367, 2005. PMID: 16125968. DOI: $10.1016 /$ j.tem.2005.08.005

17 Chen J, Yao Y, Gong C, Yu F, Su S, Chen J, Liu B, Deng H, Wang F, Lin L, Yao H, Su F, Anderson KS, Liu Q, Ewen ME, Yao X and Song E: CCL18 from tumor-associated macrophages promotes 
breast cancer metastasis via PITPNM3. Cancer Cell 19(4): 541555, 2011. PMID: 21481794. DOI: 10.1016/j.ccr.2011.02.006

18 Prasse A, Pechkovsky DV, Toews GB, Schäfer M, Eggeling S, Ludwig C, Germann M, Kollert F, Zissel G and MüllerQuernheim J: CCL18 as an indicator of pulmonary fibrotic activity in idiopathic interstitial pneumonias and systemic sclerosis. Arthritis Rheum 56(5): 1685-1693, 2007. PMID: 17469163. DOI: $10.1002 /$ art.22559

19 Lin X, Chen L, Yao Y, Zhao R, Cui X, Chen J, Hou K, Zhang M, Su F, Chen J and Song E: CCL18-mediated down-regulation of miR98 and miR27b promotes breast cancer metastasis. Oncotarget 6(24): 20485-20499, 2011. PMID: 26244871. DOI: 10.18632/oncotarget.4107

20 Leung SY, Yuen ST, Chu K-M, Mathy JA, Li R, Chan AS, Law $\mathrm{S}$, Wong J, Chen $\mathrm{X}$ and So S: Expression profiling identifies chemokine (C-C motif) ligand 18 as an independent prognostic indicator in gastric cancer. Gastroenterology 127(2): 457-469, 2004. PMID: 15300578. DOI: 10.1053/j.gastro.2004.05.031

21 Schutyser E, Struyf S, Proost P, Opdenakker G, Laureys G, Verhasselt B, Peperstraete L, Van de Putte I, Saccani A, Allavena P, Mantovani A and Van Damme J: Identification of biologically active chemokine isoforms from ascitic fluid and elevated levels of CCL18/pulmonary and activation-regulated chemokine in ovarian carcinoma. J Biol Chem 277(27): 24584-24593, 2002. PMID: 11978786. DOI: 10.1074/jbc.M112275200

22 Cai M, Bonella F, He X, Sixt SU, Sarria R, Guzman J and Costabel U: CCL18 in serum, BAL fluid and alveolar macrophage culture supernatant in interstitial lung diseases. Respir Med 107(9): 1444-1452, 2013. PMID: 23831213. DOI: 10.1016/j.rmed.2013.06.004

23 Heerboth S, Housman G, Leary M, Longacre M, Byler S, Lapinska K, Willbanks A and Sarkar S: EMT and tumor metastasis. Clin Transl Med 4: 6, 2015. PMID: 25852822. DOI: 10.1186/s40169-015-0048-3

24 Tsai JH and Yang J: Epithelial-mesenchymal plasticity in carcinoma metastasis. Genes Dev 27(20): 2192-2206, 2013. PMID: 24142872. DOI: 10.1101/gad.225334.113

25 Ying L, Chen Q, Wang Y, Zhou Z, Huang Y and Qiu F: Upregulated MALAT-1 contributes to bladder cancer cell migration by inducing epithelial-to-mesenchymal transition. Mol Biosyst 8(9): 2289-2294, 2012. PMID: 22722759. DOI: $10.1039 / \mathrm{c} 2 \mathrm{mb} 25070 \mathrm{e}$
26 Schmidt LH, Spieker T, Koschmieder S, Schäffers S, Humberg J, Jungen D, Bulk E, Hascher A, Wittmer D, Marra A, Hillejan L, Wiebe K, Berdel WE, Wiewrodt R and Muller-Tidow C: The long noncoding MALAT-1 RNA indicates a poor prognosis in non-small cell lung cancer and induces migration and tumor growth. J Thorac Oncol Off Publ Int Assoc Study Lung Cancer 6(12): 1984-1992, 2011. PMID: 22088988. DOI: 10.1097/JTO.0b013e3182307eac

27 Xu S, Sui S, Zhang J, Bai N, Shi Q, Zhang G, Gao S, You Z, Zhan C, Liu F and Pang D: Downregulation of long noncoding RNA MALAT1 induces epithelial-to-mesenchymal transition via the PI3K-AKT pathway in breast cancer. Int J Clin Exp Pathol 8(5): 4881-4891, 2015. PMID: 26191181.

28. Subik K, Lee JF, Baxter L, Strzepek T, Costello D, Crowley P, Xing L, Hung MC, Bonfiglio T, Hicks DG and Tang P: The expression patterns of ER, PR, HER2, CK5/6, EGFR, Ki-67 and AR by immunohistochemical analysis in breast cancer cell lines. Breast Cancer (Auckl) 20(4): 35-41, 2010. PMID: 20697531.

29. Hong R, Shen MH, Xie XH and Ruan SM: Inhibition of breast cancer metastasis via PITPNM3 by pachymic acid. Asian Pac J Cancer Prev 13(5): 1877-1880, 2012. PMID: 22901140. DOI: 10.7314/apjcp.2012.13.5.1877

30 Atamas SP, Luzina IG, Choi J, Tsymbalyuk N, Carbonetti NH, Singh IS, Trojanowska M, Jimenez SA and White B: Pulmonary and activation-regulated chemokine stimulates collagen production in lung fibroblasts. Am J Respir Cell Mol Biol 29(6): 743-749, 2003. PMID: 12805086. DOI: 10.1165/rcmb.2003$0078 \mathrm{OC}$

31 Zissel G, Höhne K, Kilic A, Maier C, Goldmann T, Prasse A, Ploenes $\mathrm{T}$, Trepel $\mathrm{M}$, Eibel $\mathrm{H}$ and Müller-Quernheim J: Identification of the CCL18 receptor - effects of CCL18 on human lung fibroblasts in pulmonary fibrosis are mediated via CCR6. ATS J 66, 2011. DOI: 10.1164/ajrccm-conference.2011.183.1_Meeting Abstracts.A5574

32 Legler DF and Thelen M: New insights in chemokine signaling. F1000Research 7: 95, 2018. PMID: 29416853. DOI: 10.12688/ f1000research.13130.1
Received March 8, 2020

Revised May 9, 2020

Accepted May 18, 2020 